\title{
Study of the relationship between neutrophil-to- lymphocyte ratio and parathyroid hormone levels in patients undergoing hemodialysis, Isfahan, Iran
}

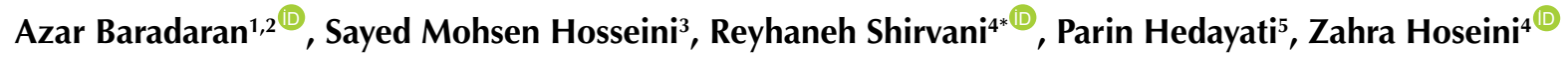 \\ ${ }^{1}$ Department of Pathology, Isfahan University of Medical Sciences, Isfahan, Iran \\ ${ }^{2}$ Infectious Disease and Tropical Medicine Research Center, Isfahan University of Medical Sciences, Isfahan, Iran \\ ${ }^{3}$ School of Nutrition and Food Science, Isfahan University of Medical Sciences, Isfahan, Iran \\ ${ }^{4}$ Faculty of Medicine, Student Research Center, Isfahan University of Medical Sciences, Isfahan, Iran \\ ${ }^{5}$ Department of Hemodialysis, Amin Hospital, Isfahan, Iran
}

Correspondence to:

Reyhane Shirvani, Email:

reihaneshirvani@rocketmail.com

Received: 11 Jan. 2021

Accepted: 31 May 2021

ePublished: 10 July 2021

Keywords: Neutrophil to lymphocyte ratio, Parathormone, Hemodialysis, Inflammation, Inflammatory mediators, Chronic kidney disease, Parathyroid hormone

\begin{abstract}
Introduction: Chronic kidney disease (CKD) is a progressive and irreversible disorder that now is introduced as one of the most important problems of general health (1). Serum levels of inflammatory mediators such as C-reactive protein (CRP), tumor necrosis factor- $\alpha$ (TNF- $\alpha$ ), interleukin-6 (IL-6) elevate in patients with endstage renal disease (ESRD). Systemic inflammation is correlated with cardiovascular disease. Furthermore, parathyroid hormone (PTH) causes releasing of IL-6 from liver and osteoblast. IL-6 increases lifetime and activity of neutrophils. Therefore, PTH may have a direct correlation with neutrophil-to-lymphocyte ratio (NLR).

Objectives: In this study, we aimed to determine the performance of NLR in hemodialysis and its possible correlation with PTH.

Patients and Methods: This is a cross-sectional study to evaluate the correlation of NLR and serum intact PTH (iPTH), Ca (calcium) and P (phosphorus) and also CaxP products $\left(\mathrm{mg}^{2} / \mathrm{dL}^{2}\right)$ in a group of stable hemodialysis patients, including male and female gender subgroups, diabetic versus non-diabetic patients, and also patients with or without chronic hypertensive disease.

Results: This study consisted of 136 hemodialysis patients. Our study showed no statistically significant correlation between iPTH (intact parathormone) and NLR in hemodialysis patients $(P>0.05)$. In addition, we test their correlation in other subgroups including hypertensive and normotensive, diabetic and nondiabetics, and male versus female patients. We found no significant correlation between iPTH and NLR in the mentioned subgroups, too.

Conclusion: Observed correlation between PTH and NLR in hemodialysis patients may be due to our small sample size. Therefore, further studies on this subject are suggested.
\end{abstract}

Introduction

Chronic kidney disease (CKD) is a progressive and irreversible disorder that now is introduced as one of the most important problems of general health. CKD is one of the most prevalent diseases in the world and Iran that the prevalence in the world is 242 per 1000 and in Iran about 1200-1600 people are added to them every year (1).

The progression of kidney disease is estimated by glomerular filtration rate (GFR). In end-stage renal disease (ESRD) the accumulation of toxins, fluid and electrolytes that normally were excreted by the kidney, caused uremic syndrome in these patients and if these toxins would not be excreted by dialysis result in their death (2). Patients

\section{Key point}

In a study on 136 hemodialysis patients, we found no statistically significant correlation between intact parathormone and neutrophil-to-lymphocyte ratio in hemodialysis patients.

with kidney disease that are undergoing hemodialysis suffer from more pathological process that most of these mechanisms are related to each other (2). In the recent decade, chronic inflammation has more importance because of the improvements of defining the pathogenesis of atherosclerosis. Serum levels of inflammatory mediators such as C-reactive protein (CRP), tumor necrosis factor-a (TNF- $\alpha$ ), interleukin-6 (IL-6) elevate in patients with end stage

Copyright $\odot 2021$ The Author(s); Published by Society of Diabetic Nephropathy Prevention. This is an open-access article distributed under the terms of the Creative Commons Attribution License (http://creativecommons.org/licenses/by/4.0), which permits unrestricted use, distribution, and reproduction in any medium, provided the original work is properly cited. 
renal disease. Systemic inflammation is correlated with cardiovascular disease (3). Kultigin et al in a cross-sectional study that involves 61 ESRD patients in 2009 introduced that inflammatory markers including CRP, TNF-a, IL-6 were significantly increased in peritoneal dialysis patients while their levels were lower in HD (hemolysis) patients (3). Additionally, neutrophil-to-lymphocyte ratio (NLR) was found to be significantly higher in peritoneal dialysis patients (3). Therefore, NLR was positively correlated with TNF-a levels (3). Additionally, they did not find any correlation between absolute neutrophil and lymphocyte counts and serum TNF- $\alpha$ and IL- 6 levels (3). NLR is a parameter that we can easily measure in CBC (complete blood cell). Therefore, it can be used as a prognostic factor for level of inflammation in CKD patients. Furthermore, parathyroid hormone (PTH) causes releasing of IL-6 from the liver and osteoblast. IL- 6 increases lifetime and activity of neutrophils (4). Therefore, PTH may have a direct correlation with NLR.

\section{Objectives}

In this study, we aimed to determine the performance of NLR for predicting the inflammation status and its possible relationship with PTH as one of the most important uremic toxins.

\section{Patients and Methods \\ Study design}

We conducted this cross-sectional study in 2020, in Iran, Isfahan, Amin and Fatemiyeh hospitals, hemodialysis departments. Blood sampling of hemodialysis patients is routinely done to check some parameters such as CBC and iPTH (intact PTH) level. We attended at the hemodialysis departments on the days of blood sampling. After taking consent from patients and considering some inclusion and exclusion criteria, finally 136 patients were selected. The inclusion criteria were; the ESRD patients who are at least 18 years old, had been on hemodialysis for at least three months, and have no special conditions such as drug or alcohol addiction (based on patient self-expression). The exclusion criteria based on their medical history and physical examination were having active infection, having malignancy or any active inflammatory disease and taking any immunosuppressive drugs. Demographic information of these patients including age, gender, duration of being on hemodialysis, and having medical history of chronic hypertension disease or diabetes mellitus were written. Then we followed up their blood test results to obtain the other needed information for this study including NLR and the serum level of iPTH.

\section{Ethical issues}

The research followed the tenets of the Declaration of Helsinki. The Ethics Committee of Isfahan University of Medical Sciences approved this study. The institutional ethical committee at Isfahan University of Medical
Sciences approved all study protocols (IR.MUI.MED. REC.1399.1104). Accordingly, written informed consent was taken from all participants before any intervention. This study was extracted from M.D., thesis of Reyhaneh Shirvani at this university (Thesis \#3991026).

\section{Statistical analysis}

We analyzed the data using SPSS software version 21. All quantitative variables followed the normal distribution. We also used the Pearson correlation coefficient for iPTH and LNR, independent t-test for binary variable, and linear regression test to predict LNR. The significance level in the present study was less than 0.05 .

\section{Results}

This cross-sectional study was conducted on 136 patients that one of them was deleted as an outlier data because of its big NLR. Eighty-two patients were male (60.3\%) and 54 of them was female (39.7\%). For age of the patients, the mean (SD) was 61.14(13.6) years. For duration of CKD, the mean (SD) was 3.607 (3.3) years. Hypertension was detected in $63.2 \%$ of the patients while diabetes prevalence was $63.2 \%$. Plasma iPTH levels were found as $508.6 \pm 528.08 \mathrm{pg} / \mathrm{mL}$ (Max; 2500, Min; $9.80 \mathrm{pg} / \mathrm{mL}$ ), while NLR was $2.52 \pm 1.81$ (Table 1). There was no significant difference between both genders regarding NLR $(t=-0.581, P=0.562)$. NLR and $\mathrm{iPTH}$ were not statistically different across diabetes mellitus and hypertension $(P>0.05$; Table 2$)$.

In Table 3, we evaluated the correlation between serum level of iPTH levels and NLR by Pearson's correlation coefficient test, which shows no statistically significant correlation between these two variables $(P>0.05)$.

Besides, we controlled the effects of calcium $(\mathrm{Ca})$ and phosphorus (P) on NLR, but there was no significant correlation, too. Additionally, we evaluated the correlation between NLR and $\mathrm{Ca} \times \mathrm{P}$ products. However, there was no significant correlation between these two parameters, too $(P>0.05)$. Moreover, we use linear regression to determine confounding factors about neutrophil to lymphocyte ratio and detected no confounding variable $(P>0.05)$. There

Table 1. Mean and standard deviation of the basic information

\begin{tabular}{|c|c|c|c|c|}
\hline Variable & Minimum & Maximum & Mean & SD \\
\hline Age $(y)$ & 25.00 & 85.00 & 61.13 & 13.60 \\
\hline Disease duration (y) & 0.20 & 30.00 & 3.60 & 3.31 \\
\hline $\mathrm{WBC} / \mu \mathrm{L}$ & 2300 & 18200 & 6822.79 & 2266.53 \\
\hline Neutrophil/ $\mu \mathrm{L}$ & 16.00 & 89.30 & 60.33 & 11.46 \\
\hline Lymphocyte/uL & 6.00 & 74.00 & 29.21 & 10.19 \\
\hline iPTH $(\mathrm{pg} / \mathrm{mL})$ & 9.80 & 2500.00 & 508.60 & 528.08 \\
\hline $\mathrm{Ca}(\mathrm{mg} / \mathrm{dL})$ & 6.30 & 14.00 & 9.02 & 1.04 \\
\hline $\mathrm{P}(\mathrm{mg} / \mathrm{dL})$ & 1.90 & 8.20 & 4.76 & 1.27 \\
\hline NLR & 0.22 & 14.77 & 2.53 & 1.80 \\
\hline
\end{tabular}

iPTH, Intact parathyroid hormone; NLR, Neutrophil to lymphocyte ratio; Ca, calcium; P, phosphorus. 
NLR in hemodialysis

Table 2. Comparison of the iPTH and NLR across gender, diabetes mellitus and hypertension

\begin{tabular}{llccc}
\hline Variable & & Mean & SD & P value \\
\hline \multirow{2}{*}{ iPTH $(\mathrm{pg} / \mathrm{mL})$} & Male & 522.3 & 548.2 & \multirow{2}{*}{0.709} \\
& Female & 478.6 & 500.1 & \\
\multirow{2}{*}{ NLR } & Male & 2.458 & 1.64 & 0.562 \\
& Female & 2.643 & 2.04 & \\
iPTH & Hypertensive & 514.6 & 538.8 & \multirow{2}{*}{0.863} \\
NLR & Normotensive & 498.3 & 514.2 & \\
& Hypertensive & 2.6 & 1.8 & 0.525 \\
iPTH $(\mathrm{pg} / \mathrm{ml})$ & Normotensive & 2.4 & 1.8 & \\
& Diabetic & 462.1 & 481.9 & \multirow{2}{*}{0.179} \\
\multirow{2}{*}{ NLR } & Non-diabetic & 588.5 & 595.8 & \\
& Diabetic & 2.5 & 1.7 & \multirow{2}{*}{0.741} \\
\hline
\end{tabular}

iPTH, Intact parathyroid hormone; NLR, Neutrophil to lymphocyte ratio.

Table 3. Pearson's correlation between NLR and other effective factors

\begin{tabular}{lll}
\hline Correlation & & NLR \\
\hline \multirow{2}{*}{ PTH $(\mathrm{pg} / \mathrm{mL})$} & Pearson's coefficient & -0.109 \\
& $P$ value & 0.208 \\
Calcium $(\mathrm{mg} / \mathrm{dL})$ & Pearson's coefficient & 0.036 \\
& $P$ value & 0.684 \\
Phosphorus $(\mathrm{mg} / \mathrm{dL})$ & Pearson's coefficient & 0.078 \\
& $P$ value & 0.374 \\
Disease duration $($ year $)$ & Pearson's coefficient & -0.025 \\
& $P$ value & 0.774 \\
Ca $\times$ P products $\left(\mathrm{mg}^{2} / \mathrm{dL}^{2}\right)$ & Pearson's coefficient & 0.097 \\
& $P$ value & 0.275 \\
\hline
\end{tabular}

iPTH, Intact parathyroid hormone; NLR, Neutrophil to lymphocyte ratio; Ca, calcium; $\mathrm{P}$, phosphorus.

was no predictor variable to estimate NLR in hemodialysis patients (Table 4).

\section{Discussion}

In our study, the association of NLR with serum iPTH, Ca, P and $\mathrm{Ca} \times \mathrm{P}$ were not significant. Study regarding the impact of parathormone on NLR is limited to an investigation by Toraman et al, on 48 males and 253 females with high PTH levels in nephrology and endocrinology outpatient clinics. They showed a significant correlation between PTH and NLR is independent of GFR (4). They showed NLR is positively correlated with serum PTH. They concluded that PTH could be a pro-inflammatory parameter independent of the degree of renal dysfunction (4).

Cho et al demonstrated that NLR could define the mortality risk in patients with severe calcific aortic stenosis (5). Ahbap et al found a significant positive correlation between NLR and CRP levels in ESRD patients (6). Li et al reported that high level of NLR is associated with more cardiovascular diseases and NLR has a direct correlation with hyperhomocystinuria in hypertensive patients (7). Secondary hyperparathyroidism is one of the first metabolic disorders in ESRD patients and more than 50\% of dialysis patients are affected by this disorder and have
Table 4. Linear regression to predict the neutrophil-to-lymphocyte ratio

\begin{tabular}{lcccc}
\hline \multirow{2}{*}{ Variable } & \multicolumn{2}{c}{$\begin{array}{c}\text { Unstandardized } \\
\text { Coefficients }\end{array}$} & $\begin{array}{c}\text { Standardized } \\
\text { Coefficients }\end{array}$ & \multirow{2}{*}{$\boldsymbol{P}$ value } \\
\cline { 2 - 4 } & B & Standard error & Beta & \\
\hline Age $(\mathrm{y})$ & 0.024 & 0.013 & 0.179 & 0.060 \\
Duration $(\mathrm{y})$ & -0.018 & 0.049 & -0.033 & 0.714 \\
\hline Diabetes & -0.467 & 0.375 & -0.125 & 0.215 \\
\hline Hypertension & 0.171 & 0.345 & 0.046 & 0.622 \\
\hline Polycystic & -0.063 & 1.343 & -0.004 & 0.963 \\
\hline Gender & 0.231 & 0.328 & 0.063 & 0.484 \\
\hline Calcium $(\mathrm{mg} / \mathrm{dL})$ & -0.027 & 0.153 & -0.016 & 0.860 \\
\hline Phosphate $(\mathrm{mg} / \mathrm{dL})$ & -0.194 & 0.125 & -0.136 & 0.124 \\
iPTH $(\mathrm{pg} / \mathrm{mL})$ & 0.000 & 0.000 & -.0112 & 0.205 \\
\hline
\end{tabular}

iPTH, Intact parathyroid hormone.

symptoms such as bone pain, joint problems, fractures, pruritus and even calcification in soft tissue such as lung, vessels and skin (8). In ESRD patients, increased level of PTH occurs due to acidosis and resistant to calcitriol, increased level of $\mathrm{P}$ and decreased level of blood calcium.

Yang et al in a study on 231 patients that were parathyroidectomy in 2010-2015 showed that NLR and PLR level decrease in these patients that shows the modulatory effect of parathyroidectomy and decrease level of PTH on systemic inflammation (9). Recent studies showed that patients with stages 3-5 CKD are not undergoing dialysis, a little increase in PTH level is correlated with increased risk of cardiovascular diseases (regardless of serum levels of calcium, phosphorous and vitamin D) (10). Therefore in these studies special attention to monitor the level of PTH from early stages of CKD and treatment of any disorder in minerals level that result in increased $\mathrm{iPTH}$ level is recommended (10). Early treatment of mineral level disorders in CKD can help the prevention of CVDs (10). Hence, there is a great interest in establishing a target range for PTH in different stages of CKD (10).

A cross-sectional study was conducted by Bhuriya et al in 4472 patients with CKD stage 3-4, demonstrated that PTH and GFR are two independent prognostic factors for CVD in CKD patients. Wonderfully, in this study there was no correlation between level of $\mathrm{Ca}$ and $\mathrm{P}$ with prevalence of CVDs (11).

Nevertheless, there are inconsistent results about the relation between PTH and level of inflammatory markers. In some studies level of inflammatory markers increased in hyperparathyroidism while in others, level of these markers was the same as control group (4). Therefore $\mathrm{PTH}$ level is not an appropriate prognostic factor for the beginning of bone turnover and inflammation level in any case and diagnostic kits have some methodological restrictions (10). Additionally the review study conducted by Pontoriero et al could not determine a special range for PTH level in CKD patients (10).

Premature cardiovascular disease is one of the most 
common causes of mortality in ESRD patients (12). This risk increases in HD patients (13). White blood cell (WBC) count and its subtypes are the classic markers for inflammation in cardiovascular disease (14). In recent years neutrophilia and related lymphopenia were introduced as independent factors for mortality in patients with acute heart disease (15-16). Additionally NLR was introduced as a potent marker for inflammation in cardiac and non-cardiac patients (17-19). In a study, DescampsLatscha et al introduced that density of many cytokines in patients with uremia increases without any relationship with dialysis and level of inflammation is higher in HD patients secondary to frequent use of dialysis membrane (20).

Cardiovascular diseases mortality in HD patients is much higher than that in general population and it is not yet explained by traditional risk factors for CVD (cardiovascular disease) (21). Micro inflammation is an important factor in the pathogenesis of CVD in HD patients and it can further accelerate the progression of atherosclerosis (22).

PTH causes releasing IL-6 from the liver and osteoblasts that increases lifetime and activity of neutrophils in addition to entrance of them into the circulation (4).

Therefore we expected high levels of PTH causes increasing the level of NLR; the same as the study by Toraman et al, which showed the positive correlation between NLR and PTH (4). But in our study there was no significant correlation between PTH and NLR in any subgroups including diabetic or non-diabetic, hypertensive or normotensive and male or female groups. Additionally we controlled the effects of $\mathrm{Ca}$ and $\mathrm{P}$ but it was not founded any correlation too.

\section{Conclusion}

Our study was the second one that was conducted on the correlation between PTH and NLR in hemodialysis patients and unlike previous study in this field, showed that there is no significant correlation between these two parameters. These results could be due to our small sample size and more researches with larger sample sizes are needed.

\section{Limitations of the study}

This study was conducted in only two departments of hemodialysis. More studies with more sample sizes specially for evaluating the effect of underlying causes of ESRD are needed.

\section{Author's contribution}

$\mathrm{AB}$ and $\mathrm{RS}$ were the principal investigators of the study. $\mathrm{ZH}$ and $\mathrm{PH}$ participated in gathering the data. SMH conducted the statistical analysis. All authors participated in preparing the final draft of the manuscript, revised the manuscript and critically evaluated the intellectual contents. All authors read and approved the content of the manuscript and confirmed the accuracy or integrity of any part of the work.

\section{Conflicts of interest}

The authors declare that they have no competing interests.

\section{Ethical considerations}

Ethical issues (including plagiarism, data fabrication, double publication) have been completely observed by the authors.

\section{Funding/support}

None.

\section{References}

1. Ammirati AL. Chronic Kidney Disease. Rev Assoc Med Bras (1992). 2020; 66 Suppl 1:s03-9. doi: 10.1590/1806-9282.66. S1.3.

2. Nigam SK, Bush KT. Uraemic syndrome of chronic kidney disease: altered remote sensing and signalling. Nat Rev Nephrol. 2019;15:301-16. doi: 10.1038/s41581-019-0111-1.

3. Turkmen K, Guney I, Yerlikaya FH, Tonbul HZ. The relationship between neutrophil-to-lymphocyte ratio and inflammation in end-stage renal disease patients. Ren Fail. 2012;34:155-9. doi: 10.3109/0886022X.2011.641514.

4. Toraman A, Aras F, Hekimsoy Z, Kursat S. Is there a relationship between parathyroid hormone and neutrophil lymphocyte ratio or platelet lymphocyte ratio? Acta Endocrinol (Buchar). 2019;5:96-101. doi: 10.4183/aeb.2019.96.

5. Cho KI, Cho SH, Her AY, Singh GB, Shin ES. Prognostic Utility of Neutrophil-to-Lymphocyte Ratio on Adverse Clinical Outcomes in Patients with Severe Calcific Aortic Stenosis. PLoS One. 2016;11:e0161530. doi: 10.1371/journal. pone.0161530.

6. Ahbap E, Sakaci T, Kara E, Sahutoglu T, Koc Y, Basturk T, et al. Neutrophil-to-lymphocyte ratio and platelet-tolymphocyte ratio in evaluation of inflammation in end-stage renal disease. Clin Nephrol. 2016;85:199-208. doi: 10.5414/CN108584.

7. Li H, Lu X, Xiong R, Wang S. High neutrophil-to-lymphocyte ratio predicts cardiovascular mortality in chronic hemodialysis patients. Mediators Inflamm. 2017;2017:9327136. doi: 10.1155/2017/9327136.

8. Rodríguez-Ortiz ME, Rodríguez M. Recent advances in understanding and managing secondary hyperparathyroidism in chronic kidney disease. F1000Res. 2020;9:F1000 Faculty Rev-1077. doi: 10.12688/f1000research.22636.1.

9. Yang PS, Liu CL, Liu TP, Chen $\mathrm{HH}, \mathrm{Wu} \mathrm{CJ}$, Cheng SP. Parathyroidectomy decreases neutrophil-to-lymphocyte and platelet-to-lymphocyte ratios. J Surg Res. 2018;224:169-75. doi: 10.1016/j.jss.2017.12.016.

10. Pontoriero G, Cozzolino M, Locatelli F, Brancaccio D. CKD patients: the dilemma of serum PTH levels. Nephron Clin Pract. 2010;116:c263-8. doi: 10.1159/000318787.

11. Bhuriya R, Li S, Chen SC, McCullough PA, Bakris GL. Plasma parathyroid hormone level and prevalent cardiovascular disease in CKD stages 3 and 4: an analysis from the Kidney Early Evaluation Program (KEEP). Am J Kidney Dis. 2009;53:S310. doi: 10.1053/j.ajkd.2008.11.029.

12. Johnston $N$, Dargie $H$, Jardine A. Diagnosis and treatment of coronary artery disease in patients with chronic kidney disease: Ischaemic heart disease. Heart. 2008;94:1080-8. doi: 10.1136/hrt.2007.136739.

13. Collins AJ. Cardiovascular mortality in end-stage renal disease. Am J Med Sci. 2003;325:163-7. doi: 10.1097/00000441200304000-00002.

14. Horne BD, Anderson JL, John JM, Weaver A, Bair TL, Jensen KR, et al. Intermountain Heart Collaborative Study Group. Which white blood cell subtypes predict increased cardiovascular risk? J Am Coll Cardiol. 2005;45:1638-43. doi: 10.1016/j. jacc.2005.02.054 
15. Arruda-Olson AM, Reeder GS, Bell MR, Weston SA, Roger VL. Neutrophilia predicts death and heart failure after myocardial infarction: a community-based study. Circ Cardiovasc Qual Outcomes. 2009;2:656-62. doi: 10.1161/ CIRCOUTCOMES.108.831024.

16. Rudiger A, Burckhardt OA, Harpes P, Müller SA, Follath F. The relative lymphocyte count on hospital admission is a risk factor for long-term mortality in patients with acute heart failure. Am J Emerg Med. 2006;24:451-4. doi: 10.1016/j. ajem.2005.10.010.

17. Tamhane UU, Aneja S, Montgomery D, Rogers EK, Eagle KA, Gurm HS. Association between admission neutrophil to lymphocyte ratio and outcomes in patients with acute coronary syndrome. Am J Cardiol. 2008;102:653-7. doi: 10.1016/j. amjcard.2008.05.006.

18. Núñez J, Núñez E, Bodí V, Sanchis J, Miñana G, Mainar L, et al. Usefulness of the neutrophil to lymphocyte ratio in predicting long-term mortality in ST segment elevation myocardial infarction. Am J Cardiol. 2008;101:747-52. doi: 10.1016/j. amjcard.2007.11.004.
19. Walsh SR, Cook EJ, Goulder F, Justin TA, Keeling NJ. Neutrophillymphocyte ratio as a prognostic factor in colorectal cancer. J Surg Oncol. 2005;91:181-4. doi: 10.1002/jso.20329. PMID: 16118772.

20. Descamps-Latscha B, Herbelin A, Nguyen AT, Roux-Lombard P, Zingraff J, Moynot A, , et al. Balance between IL-1 beta, TNFalpha, and their specific inhibitors in chronic renal failure and maintenance dialysis. Relationships with activation markers of T cells, B cells, and monocytes. J Immunol. 1995;154:882-92.

21. Tonelli M, Karumanchi SA, Thadhani R. Epidemiology and Mechanisms of Uremia-Related Cardiovascular Disease. Circulation. 2016;133(5):518-36. doi: 10.1161/ CIRCULATIONAHA.115.018713. Erratum in: Circulation. 2016 Mar 8;133(10):e435.

22. Neirynck N, Glorieux G, Schepers E, Dhondt A, Verbeke F, Vanholder R. Pro-inflammatory cytokines and leukocyte oxidative burst in chronic kidney disease: culprits or innocent bystanders? Nephrol Dial Transplant. 2015;30:943-51. doi: 10.1093/ndt/gfu409. 